\title{
Ciudadanos y siervos El "hombre mecánico", la justicia y la democracia
}

\author{
- Froncisco Fernandez Buey
}

\section{Preguntas para el mundo actual}

1.1. Una teoría no procedimental o formalista de la democracia y de la justicia en el mundo actual tiene que tener en cuenta al menos las dos preguntas siguientes:

- ¿Qué significa ser ciudadano en un mundo dominado por la lecnologia?

- ¿Hasta qué punto es realizable el ideal clisico, aristolélico y republicano, del zoon politikon en la épocal de la robólica, de la cibernélica, de la informílica, de la computación, de la lelemática y de la inteligencia arlificial?"

- ¿Siguen sirviendo los viejos valores de la justicia y de la democracia, heredados de la cultura griega, del Renacimiento y de la llusiracion, en un mundo globalizado en el que la aulomatización y la compulación reemplazan a rilmo acelerado el trabajo manual humano y parecen apuntar hacia un fuluro "poshiológico" y "sobrenalural”."

Hans Moravec, investigador en el campo de la robótical y director del laboratorio de rohots móviles de la Universidad (arnegie Mellon. en un libro publicaldo por las prensas universilarias de Harvard a principios de esta decada, ha escrito que nos aproximamos al momento en que prácticamente lodas las funciones humanas esenciales, físicals y mentales, lendrín su equivalente artificial, y que el prototipo de la unión de todos los resultados del desarrollo cultural será el rohot inteligente, una máquina que podrá pensar y actuar como un ser humano. aunque física y moralmente no lo seal. Esto fue escrito en $1 \%()$ antes de que se 
revelara la posibilidad de la clonación de humanos y con independencia de las investigaciones que se llevaban a cabo en el campo de la ingenierial genétical.

En 1998 se puede pensar. por lanto, que no está lejos el momento en que la combinación de la clonación de humanos por ingeniería genélical y los avances en el campo de la robótica y de la inteligencia artificial permirá la creación de quimeras del lipo de los "replicantes" que aparecian en la pelicula futurista Blade Runner. Moravec piensal que se esti produciendo un relever génético, algo así como una mutación antropológica, y que, cuando éste hayal acabado de producirse, nuestral cultura podri evolucionar con independencia de la hiologia humana y de sus limilaciones. La evolución de nuestra cultura seguiría asi, en esta nueva fase que se está abriendo, la líneal expansiva que ha sido característica de los últimos siglos. lil paso siguiente. que en cierto modo, continuría la lendencia expansiva puesta de manifiesto desde el siglo XVI, desde el siglo de los grandes descubrimientos geográficos y de la expansión en el planeta Tierra, tendria que ser la expansión al cosmos del hombre modificado: la odiseal del espacio en el sentido de Kubrick.

Fste es un escenario futurista analiticamente razonable por lo (jue sabemos yal i. Lo es lambién, es también razonahle, desde el punto de vista de la lïlosolïa moral y política? ¿No tiene ese escenario consecuencials y ricsgos muy negativos paral el género humano?

Estil preguntal admite diferentes respuestas, lambién ralzonadas, que conviene discutir. Empecemos por la exposición de la "lecnofohia" y de la "tecnolilia".

|"Nuestros inventos no son más que medios mejorados para un lin que no mejoril" (Thorcau-Freud)).

La respuesta tecnófoba más radical de las expresiadas hastal este momento la hal dado Theodore Kaczynsky, presunto autor de una serie de alentados en USA firmados con el nombre de Unahomber y se puede leer en Internet en el Manifiesto que hizo circular unos meses antes de su detención. Kalczynsky es un matemático con un excelente historial profesional en varials de las más prestigiosals universidades americanas convertido en "terrorista científico". ^ partir de un determinado momento el objetivo de sus actos terroristas han sido instituciones y personas del estahlishment tecnocientifico.

En su Manifiesı, que tiene 232 puntos, Kaczynsky, parte de la idea de que los males de nuestra sociedad provienen de la tecnología: "La revolución industrial y sus consecuencias han sido un desastre para el género humano". Analiza luego los efectos negativos, en el plano sociopolítico, del sistema industrial-tecnológico: critica las más importantes manifestaciones del izquierdismo y de la "corección política" en el sistema norteamericano actual poniendo el acento en el análisis de la "sensación de inferioridad" (10-23), denuncial el carácter subalterno (38 y ss.) de la mayoría de las actividades de la izquierda que se centran sólo en 
el problema social. Considera que la ciencia y la lecnologia acuales constiluyen el principal ejemplo de actividades subordinadas, de carícler subalterno (87) y denuncia la ideología cientilista cue presenta las aclividades del complejo tecnocientílico como un benelicio para la humanidad (88). Propugna una revolución cuyo objelivo no sería derribar a los gobicrnos sino la basce económca y tecnlógica de la sociedad actual. Ea conjunto es un incpuictante alegalo de lipo luddila que conviene conocer en loda sil extension.

Si el Manifiesto de Kalc\%ynsky es priclicamente desconocido (soblo he visto una referencia a él en un arliculo de Pere Puigdomènech publicado hace unos meses en l:l País), en cambio, si ha sido objeto de mucha alencion el libro de Neil Postman, Teconópolis, publicado en casiclano, en ley)4, por el ("irculo de Lectores. Su subtitulo, La rendición de la cultura a la leconologica es igualmente significativo, pero pueda dar lugar a ecpuivocos, puesio cue el autor, peclagogo y teórico de la comunicación norteamericano, no es un leconolobo, sino alguien que busca y propugna una tereera cultura frente a Teconopolis. In una direccion, precisamente, muy próxima a la que intentamos seguir en esta lacullad de Ilamanidades. El libro concluye justamente con una propuesta pedageggica en la que se da mucha imporlancia a la historia y a la libosolia de la ciencia. a la semántica (como ayuda para detectar trolas en la sociedacl de la comunicacion y de la publicidad), a la historia del arte (como edacacion de los scontimientos y de la sensibilidad, demo forma de educacion sentimental), al estudio de las religiones comparadas: "Ninguna educacion puede rechazar lextos sagrados como el Génesis, el Nuevo Testamento, el (orín o el Bhagavad-( iila. (adla uno de ellos encarna un estilo y una imagen del mundo que nos dice lanto sobre el ascenso de la humanidad como colalguier otro libro escrito. $\Lambda$ esos libros aniadiria el Manifiesto comunista, pues pienso (fuc es razonable calilicarlo como un lexto sagrado, que encarna principios religiosos a los que muy recientemente millones de personas han sido (levotos" ( de. cil. 254).

Ilay un par de ideas en el libro de Posiman cue conviene resallar:

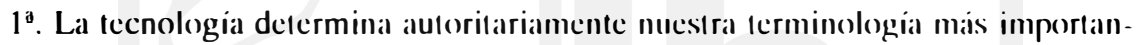
te; redefine "liberlad", "verdad", "inteligencia", "sabicluria", "memoria", "historia".

2a. Toda tecnología tiene dos caras. Pero no sólo liene dos caras sino yue en su imposición hay ganadores y perdedores, benelicios y perjuicios que no se reparten equitativamente. Un ejemplo. El caso del reloj mecánico, que tuvo su origen en los monasterios benediclinos del siglo XII y XIII, con sicte periodos de oración durante el dia y que podía proporcionar devoción a esos rituales de devoción, se convierte luego en el elemento de regulación y uniformización de la vida del trabajador para los productores estandarizados, de la mayor utilidad para hombres que que descahan dedicarse a la acumulación de dinero. De Dios a Mammón, el dios de la riqueza. En medio hay un

Cludadanos y siervos: El "hombre mecánico", la justicia y la democracia 
momento (1370) en que el rey Carlos V de Francia ordenó a todos los ciudadanos de Paris que regularan su vida privada, comercial e industrial, según las campanas del reloj del Palacio Real, que sonaban cada sesenta minulos.

1.2. La segunda pregunta se puede lormular así: ¿Qué quiere decir ser ciudadano en una plétora miserable en la que casi dos tercios de la población mundial vive en la pobreza, millones de personas siguen siendo tratados literalmente como esclavos y en muchísimos paises de Asia, Alrica, Lalinoamérica y Luropa el sulragio universal está resiringido por hechos como la discriminación sociocultural de las mujeres y porque muchas veces se vola, cuando se vola, bajo las imposiciones de los ejércitos, o la intervención directa de éstos, y con la tolerancia de las grandes potencias económicas que se autodefinen como "democracias liberales"? Desarrollo de esie punto en F. Fernández. Bucy/J. Riechmann, Ni trihunos. Madrid, Siglo XXI, 1996, cap. 2.: "Crílica de una democracia demediada"].

El punto de vista procedimentalista of formalista sobre democracia y justicia suele admitir, por activa o por pasiva, el tópico de que el liberalismo democrático, o la democracia liberal, es hoy la forma más extendida de gobernar en el mundo; define luego formalmente lo que son las reglas del juego de la democracia liberal (sufragio universal, división de poderes, existencia de un parlamento, existencia de una carta constitucional mayorilariamente aprobada y alternancia en la gobernación); observa después que, en la práctica, existen ciertos déficits en relación con estos rasgos o caracteristicas en las sociedades de nuestro marco cultural; argumenta que esos déficits no son nada en comparación con los despotismos y tiranías que han existido en otros momentos y en olras parles del mundo; y suele concluir con una seric de consideraciones tan bienintencionadas como etnocéntricas referidas ya exclusivamente, por lo general, a la democracia liberal, en singular, como el "mejor de los mundos posibles" o el "menos malo de los mundos reales" [Tal es punto de visla que se desarrolla en publicaciones recientes de Francis Fukuyama y de Samuel (). Huntingın, por ejemplol.

\section{A esta forma de argumentar se han opuesto ohjeciones de distinto tenor:}

2.1. Una primera objeción es de carácter analílico y se puede expresar como sigue. El lópico, muy extendido, según el cual la democracia liberal o el liberalismo democrático es la lorma más extendida de gohernar en el mundo aclual es un contrafáctico. Si se hojean los informes anuales sobre el estado del mundo publicados por instiluciones internacionales independientes, o los inlormes de Amnistia Internacional para estos úlimos años, se da uno cuenta de hasta qué punio es patente la contradicción entre lo dieen las teorías formales de la democracia y lo que hay en el mundo a esie respecto. Para una crílica desmitilicadora de lo que ha sido la "democracia" realmente existente en la últimas décadas: $N$. Chomsky, El miedo a la democracio. Barcelona. Crítica, 19921.

\section{4}

Realidad 83, 2001 
2.2. Una segunda objecion se hasal en consideraciones historicals sobre el concepto mismo de democracial. I lahbando con propiedald, fue es como hay que intentar hablar, "(lemocracial" en el senticlo pleno de lo gue dice literalmente esta palabra ("gohicrno (lel puchlo"), no ha hahido hasial ahora nuncal en el mundo. La democracia, así entendida, sigue siendo un ideal.

I n la Atenas del siglo $V$ antes de nuestra era, que es donde nacio lo que se ha llamado "democracia", el gohierno del de'mos estahal resiringido por el derecho de ciudadania y éste dejaba fuera de consideracion a las mujeres y a los esclavos (la mayoría absoluta de la poblacion). J ' $f$. a este respecto Ste. (roix,

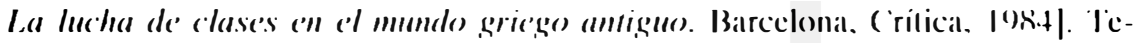
nia, en cambio, la particularidad de ser una democracia directa. alsamblearial en la que los miemhres de la polis con derecho de ciudadania se reunian y votaban las grandes decisiones (jue alectaban a su repriblical Pero. por olral parle, ya Aristóteles, en el libro sexto de la l'olitica, Ilamaba la allencion acerea del hecho de que la "democracia" no soilo se decial en (irecia de muchas maneras sino que habia diferentes tipos de "democracia" en funcion de las combinaciones entre las diferentes culturats y estrallos sociales.

Fista consideración vale igualmente para la llamada "democracia" medieval y para la democracia "moderna". De hecho lass repúblicass llamaldas " (lemocrailicas", como Venecia o Iilorencia en los alloores de la lidad Moderna, o los cantones suizos ya en la época moderna, conservaron la ideal de la democracia direcla, pero el memero de las personas que formalmente podian parlicipar en la administración y gohernacion de la cosal publica era en ella reducido. Asi es que si entendemos por "puchlo" la mayoria de los habilantes de un lerrilorio (ciudad. municipio, nación, estado, ele.) lodlas lats democracias gue han existiclo con ese nombre hasta ahora han sido democracials restingidals. Incluso si entendemos por gohierno no ya la posibilidald de volar y decidir directamente sobre los grandes asuntos de la ciudad, de la nación o de la estado, sino la posibilidad de volar para elegir a los que gohernarán realmente, haly que tener en cuenta que este (lerecho soblo se ha hecho universial muy recientemente y soiko en algunos paises del mundo. I a democracia Ilamada "indirecla" o "representalival" dejo fuera (lurante mucho liempo al cuarlo estado, a las mujeres, a los jovenes menores de 21 años. elc. Todavia ahora está en discusión en muchos paises de la Union Europea si los inmigrantes no regularizados han de lener o no derecho al volo. [Sobre este punto: Javier de I ucals, P'uerlas que se cierran. Batrcelona, Läri, 19961 .

2.3. Cuando la objeción hasalda en consideraciones históricass se une a la consideración analítica suele concluir en la idea de fue la democracia, loda democracia, es siempre un proceso en construcción. I a ampliación de los derechos de ciudadanía hasta llegar al sufragio universal ha sido una larguísima lucha en la historia de nuestro marco cultural. Este punto de vistal esila muy 
presente en algunas teorías contemporáneas de la democracia, que tratan de conjugar libertad, justicia e igualdad, como, por ejemplo, la de Norberto Bobhio. Y en tal șentido tiene razón el escritor John Berger cuando afirmaba hace poco que "la democracia no es un sistema, como falsamente se enseña, sino una forma de resistencia que va cambiando continuamente a lo largo de la historia"[Cf. Páginas de la herida. Madrid, Visor, 19961.

2.4. Una cuarta objeción afirma que la teoría formal o procedimental de la democracia contemporánea tiende a hacer abstracción de lo que habría que llamar la "constitución material", es decir, de las consecuencias que tiene en el plano político la persistencia de las diferencias sociocconómicas. Estas limitan materialmente no sólo la participación de buena parte de la ciudadanía con derechos en los asuntos de la polis sino también la representación política misma. De hecho, por debajo de lo que dicen las Constituciones o cartas constituciones en las democracias liberales representativas, la "constitución material" contradice la igualdad formal ante la ley y conduce a que unos estratos sociales, los más altos, estén sobrerrepresentados mientras que otros, los más bajos, estén minusrepresentados. [Un desarrollo de este punto de vista en: J. R. Capella, Ios ciudadanos sicrvos. Madrid, Trotta, 1994, cap. II].

2.5. Una quinta objeción argumenta que la imparable lecnificación del ámbito polílico y la mercantilización de la actividad de los partidos (en gran parte dependientes de los créditos hancarios privados y/o de la linanciación estatal) son factores que contribuyen a "demediar" las democracias realmente existentes ya en el ámbito de los procesos electorales. De manera que podría decirse (con Icibniz y con Lessing) que si el mal menor resulta ser un hicn, cl hien menor resulta ser un mal. La percepción de la democracia realmenle exislente como un "bien menor" suele derivar en "melancolía democrílica". Por eso algunos autores que introducen la consideración socioeconómica en el análisis de la democracia y en la redefinición de lo que es hoy la ciudadanía se preguntan con razón si las elecciones, los procesos electorales (una vez admitido formalmente el sulragio universal) son ya, sin más, la democracia (Se puede ver a este respecto la obra del economista Serge-Christophe Kolm: Les élections sont-elles la démocratice? París, Editions du Cerl,; y la discusion de ésle y otros puntos de vista en: J. M. González y F. Ouesada (Coor.), Teorias de la democracia. BarceIona, Anthropos, 1988|.

\section{El problema de la justicia}

3.I I lay, como sabe, varias acepciones del término justicia. De las varias acepciones del término "justicia” la que nos motiva mayormente, es sobre todo la jurídico-política: o sea, la que ha dado origen a la controversia acerca del tipo de organización de que dispone (o puede disponer) el Estado para reprimir y castigar los delitos y dirimir las diferencias entre los ciudadanos de acuerdo con la 
ley y el derecho. Es difícil, sin embargo, prescindir, al menos en el análisis, de otras acepciones de justicia vigentes en el lenguaje corriente. En parlicular es difícil prescindir de la acepción élica de la justicia. Pues, antes o después, loda discusión acerca del funcionámiento concreto del sistema judicial en un país determinado suele entrar (o por lo menos hordear) en el tema de las normas y de los supuestos morales.

3.2. Todavía ahora aparece recurrentemente en nuestras sociedades la idea, griega, de que algo es justo cuando su existencia no interficre con el orden al que pertenece; de modo que se define la justicia, por la negativa, como restauración o restablecimiento de un orden que se intuye natural, originario, o bien como corrección o castigo de la desmesura, de la hybris (nosotros ahora decimos casi siempre: del "desequilibrio psíquico" o de la "enaljenación mental transitoria”, pero, ¿no era la hybris precisamente eso: "enajenación mental, más o menos transitoria, del animal racional entendido como zoon politikon??).

Esla noción originariamente griega de la justicia en tanto gue corrección de la hybris está direclamente emparentada con lo (jue Ilamamos justicia "conmutativa", que es, precisamente, correctival o rectificalloria en la medicla en cjue regula las relaciones tanto voluntarias como involuntarias de unos ciudardanos con otros. Es interesante a este respecto hacer observar (fue el pensimiento griego puso siempre en comunicación directa la justicia, en tanto (jue virlud muy principal, con la felicidad. Miss aún: ser justo es, para este universo mental, algo así como una condición sine qua nom para ser feliz., para (fue la persona pueda ser feliz: y una sociedad en la cue rige la vida huena es sustancialmente acjuella en la cual, por encima de otrats cosals, reina la justicia.

Todavia hoy el lenguaje popular sucle identilicar el ser justo con el equilibrio de la persona en el juicio y en el trato de los otros; de modo (pue en el plano personal o individual justicia fue y sigue siendo, sobre todo, efpuilibrio en el juicio moral, crítica de la desmesura, consciencia de (jue, siendo el hombre todo extremos, cabe, no obstante, un comparlamiento ecuinime para con los otros miembros de la especie. En el plano social, en cambio, la aspiración a la justicia como equidad ha preferido diversos principios que (haim Perelman reduce a seis:1) $\wedge$ todos lo mismo; 2) $\wedge$ carla cual según sus méritos; 3) $\wedge$ carla cual según sus obras; 4) $\wedge$ cada cual según sus necesidades; 5) ^ cada uno según su rango; 6) $\wedge$ cada uno según lo atrihuido por la ley.

3.3. En la democracia "realmente existente" o "democracia demediatla" son muchas las personas que lienen la impresion de cue "no hay justicia", de gue se aplical la justicia con diferentes raseress e incluso (pue hay personas (pue, por su posición económical, social o política se sitúan y son siluadas "mis allii de la justicia". La controversia pública sobre la corrupción vinculada al dominio polílico (el uso y aprovechamiento privaldo de lo público) liene mucho (jue ver con eso. Pues no pocas veces se da por supuesto (o se aclúa como si se diera por 
supuesto) que los amigos ("Ios nuestros", en el sentido moral y político) quedan o deben quedar, efectivamente, más allá de la justicia.

Vale la pena detenerse en un interesante supuesto, que aparece ya en la Ética nicomaquea aristotélica; un supuesto en el cual no hace falta justicia: cuando los hombres son amigos. Esto quiere decir que la philia o la fraternidad está en cierto modo, como virtud moral propia de la persona humana, por encima de la justicia, más allá de la justicia. Se podría añadir, para precisar esta idea, que la comunidad de los amigos fraternos tiene su base en la benevolencia, en la caridad, en la piedad, en la compasión, mientras que, en cambio, la justicia, en particular la justicia conmutativa, es elemento fundamental o sustancial para la organización de una sociedad en la que se ha rebasado ya el marco psicosocial de la fraternidad, de la amistad entre iguales.

La distinción no es baladí, puesto que a pesar del tiempo transcurrido desde que las sociedades de nuestra cultura se organizaron políticamente como estados de derecho se sigue confundiendo, casi siempre aposta, a propósito, el valor moral personal, individual, de la filia o de la comprensión simpatética entre hermanos o parientes próximos con el valor ético-político de la justicia conmutativa. Esta confusión de planos ha conducido a comportamientos que pueden llegar a ser aberrantes en nuesiras sociedades. Pues, en nombre de la philia, de la fraternidad, de la caridad o de la misericordia, el padre tenderá a defender al hijo y el amigo hará valer el mismo principio moral. que está más allá de la justicia, para defender al amigo contra los juc'ées.

3.4. El pensamiento moderno, al dar cuenta del paso de la antigua comunidad a la sociedad del estado-nación, ha tendido a identificar justicia con utilidad pública, de modo que, según esto, la auténtica justicia distributiva seria la derivación de aquello que se hace en conformidad con el interés de todos (o la mayoría de) los miembros de la sociedad, mientras que la justicia conmutativa, al dar prioridad también a la utilidad publica, obliga, por asi decirlo, a poner entre paréntesis, cuando se ve una causa judicial, aquel olro valor moral derivado de la comunidad fraterna que estaba más allí de la juslicia. Pero, consciente de la importancia que así cobra la justicia en la sociedad, el pensamiento moderno, desde Montesquieu, ha querido dejar bien clara la separación entre poder judicial, legislativo y ejecutivo.

Ahora bien, el hecho de que la ordenación juridica vigente en los estados democráticos de derecho haya obligado a distinguir los planos y, por lanlo, a poner entre paréntesis, en el lado de lo privado, aquellos valores morales que essán más allá de la jussticia no resuclve o liquida por completo, como es natural, la tensión entre el comportamiento moral individual hasado lal vez en el sentimiento de la fraternidad o de la caridad y la aplicación igualitaria, ecpuitativa, de la justicia. Ia tensión entre valores morales que están más allí de la 
justicia y el ejercicio mismo de la justicia no ha cesado de crecer. Y eso se nola en la vida cotidiana. Hay que dar, por tanto, una explicación de esta tensión.

Primero debe tenerse en cuenta que el pensamiento moderno, en la cultura curoamericana, es heredero por igual de las Iradiciones grecorromana y cristiana. El choque, cruce, complementación o fusión de estas dos Iradiciones en el tema que nos ocupa ha tenido como consecuencia el que se mantuviera cierla tensión acerca de la primacia ético-politico de los valores de la justicia (generalmente equiparados al equilibrio moral y a la equidad en la marco de la socicdad ampliada) y de la filia o de la caridad (generalmente equiparados a la amislad y la fraternidad en el marco de la familia ampliada o de la comunidad). Todavía actualmente, al Iratar de la justicia (tanto en el sentido amplio como en el sentido más restringido de "tipo de organización estatal para la corrección de la injusticia"), la pareja amigo/enemigo suele acompañar las más (le veces, para competir con ella, a las categorias definitorias de las instituciones judiciales que, en el mundo contemporineo, suclen ser neutralidad, ecuanimidad, imparcialidad, objetividad.

Pero en segundo lugar hay que tener en cuenta otro dato histórico: la tradicional vinculación del sistema de justicial al poder económico, social o político, sobre lodo en los paises latinos, cosa que conlleva una persistente desconlianza en la justicia instilucionalmente organizada y en los jueces en particular. Muestras de esa desconlianza inundan nuestra literatura desde el llamado Siglo de Oro.

3.5 La consideración de que la virlud de la amistad esta más allí de la justicia sucle traducirse en nuestra sociedad en la afirmación (no siempre explicila pero casi siempre sentida) de que, en general, la amistad esta por concima de la justicia. El lenguaje administraltivo corriente en los paises lallinos ha heredado, a veces en una forma zafia, aquella huena idea aristotélica de que donde hay philica, amistad o fraterniclad no es necesaria justicia, o sea, aplicación imparcial o neutra de las leyes. In el mareo privado de la pareja, la lamilia o la comunidad fraterna más o menos restringida, sigue estando aceptada una forma de resolución de conflictos entre los humanos que permite considerar justas a personas bondadosas o caritativas sin necesidad de someterse a los tribunales de justicia.

Pero al mismo liempo sigue siendo frecuente en nuestras sociedades la ampliación indiscriminada de esta convención sociocultural a otros planos de la vida pública. Así se dice muy a menudo en el lenguaje administrativo (que incluye los establecimientos de justicia ): "A los amigos lodo; a los enemigos nada: y a los indiferentes la legislación vigente". Fs importante destacar aquí que esta zafiedad no sólo se dice habitualmente sino que, ademis, se practica constantemente. Y más aún cuando se tralia de administrar justicial.

Ciudodonos y siervos: El "hombre mecónico", lo justicio y lo democrocio 
Ocurre, pues, que lo que en origen fue una idea razonable (esta idea de que la filia y la fratemidad no necesitan de la justicia en la comunidad) ha acabado convirtiéndose, en nuestas sociedades, en una coartada peligrosa y hasla aberante, la cual, sin embargo, cuenta con muchos defensores. No hay que sorprenderse demasiado del extraño camino seguido por aquella buena idea. Ouien haya leido la parábola dostoievskiana del gran inquisidor estará al cabo de la calle en lo que hace a los resortes psicosociales que convierten un ideal en pura porquería.

Un ejemplo relativamene reciente de la vigencia de esta tensión entre justicia como equidad, en el plano jurídico-político, y valor moral de la amistad y de la fraternidad en el marco de la comunidad restringida es la inquictante alracción que ejerce sobre el espectador el planteamiento de Francis Coppola en lil l'adrino III. ¿No está esta atracción emparentada con la simpatía por el comunitarismo en una sociedad jurídico-política dominada por el sistema de partidos y que navega, entre la euforia y la dificultad, por "las gćlidas aguas del cálculo cgoísta"?

3.6. De hecho, la mayoría de las reglas y normas que hemos ido eslableciendo en el ordenamiento jurídico de los estados modernos son en el fonclo obstáculos limitadores de cierta tendencia, al parecer muy extendida, a favorecer a los nuestros, en nombre de la philía, de la fraternidad o de la caridad, por cencima de lo que es justo: si la justicia es dar al otro lo que se le debe, la caridad (o la misericordia) es precisamente dar al otro más de lo que se debe. Pero el hecho de dar al amigo, en nombre de la fraternidad, más de lo que se debe scrá considerado generalmente por la mayoría de los que son tratados juslamente, sólo como se debe, como si fuera un agravio comparativo.

Tal agravio puede ser muy grande o puede ser muy pequeño cualitativamente en una comunidad reducida, en el ágora de la ciudad-estado a la gricga. Pero se convierte en un elemento diferenciador de gran importancia cuantitativa en nuestras sociedades contemporáneas, pues el trato preferencial dado a los que mandan (económica o políticamente) por la administración de justicia, incluso antes de la fase decisoria, o sea, ya en la fase de instrucción de tal o cual causa, implica un uso tan discriminatorio de la misma como evidente para ciudadanos a los que se equipara a la hora de pagar impuestos.

En las sociedades actuales los vínculos de sangre y los lazos familiares van siendo sustituidos progresivamente por vínculos profesionales y por lazos políticos derivados de la pertenencia a un cuerpo profesional o a una asociación política. Es lógico que, siendo esto así, la afirmación de los valores morales que están más allá de la justicia se haya ido desplazando hacia la partiocular forma de "amistad" y de "fraternidad" interesada que son las corporaciones profesionales y los partidos políticos. La justicia como equidad, la igualdad ante la ley, choque entonces con la defensa corporativa o política de los nuestros. 
Esta defensa corporaliva o política de los nuestros tiende a presentar en público como afirmación de valores morales que de hecho tendemos a considerar superiores al valor de la justicia: esto es, valores que no sólo están más allá de la justicia, sino que están por encima de la justicia. El consenso gencralizado acerca del "hágase justicia igual para lodos" (y "mejore el mundo", naluralmente) se debilita entonces para excepluar del mismo a los nucstros, o sea, a los colegas de la profesión o los comilitones del partido, por razones de "amislad" y de "farternidad".

Estas actitudes partidistas o corporativas toman en la sociedad actual múltiples formas: desde la obstrucción de la justicia hasta la defensa cínica de la corrupción de los amigos del cuerpo o del partido. Un ejemplo reciente, tan llamativo como lacerante, de esio es la aptitud que ha adoptado una parte del aparato del PSOE en el llamado "caso (i^L”, cuyas forman más aberrantes han sido la carta de los "amigos socialistas" en defensa de Barrionuevo o la chapa que dice "Yo también soy Barrionuevo". Pero tampoco es este el único ejemplo. La misma valoración instrumental, politicista o corporativa, de la amistad (politica) por encima de la justicia se ha dado en la mayoría de los casos de corrupción en el uso de fondos públicos que han llegado en España hasta los tribunales.

Se puede concluir, por lanto, que la instilucionalización de la justicia como equidad tiene que hacer frente ahora a dos obstáculos paralelos: el corporativismo de los jueces y el politicismo del sistema de partidos. Ambos obstículos resultan particularmente peligrosos cuando, como sucle ocurrir, se ven reforzados por la presencia al fondo - tan duramente denunciada por Gracián- de don Dinero y sus parientes.

\section{Conclusiones}

Las objeciones manifestadas en el apartado 2 y las consideraciones sobre justicia en el 3 apuntan en una misma dirección: la crítica de la insuficiencia o limitación de las consecuencias de la filosofía política liberal más extendida. Pero incluso cuando se acepta el marco teórico del liberalismo político, en los términos en que ha sido formulado por J. Rawls en Teoría de la justicia, quedan varios problemas interesantes por discutir al tratar de democracia y de justicia como equidad. Uno de esos problemas, denunciado en la última década por autores comunitaristas, es el del hiato entre ética privada y política liberal. El propio Rawls Io ha abordado en El liheralismo político |1993] (traducción castellana: Barcelona, Crítica, 1996).

Una interesante tentativa teórica reciente de cerrar ese hiato es el libro de $\mathbf{R}$. Dworkin, Élica privada e igualitarismo político. Barcelona, Paidós, 1993. Se puede leer esia tentativa como un notable esfuerzo teórico por recuperar la inspiración original de la filosofía moral y polílica del liberalismo europeo y, en

Ciudadanos y siervos: El "hombre mecánico", la justicio y lo democracio 
ese sentido, como una forma de enlizar la Iradición liberal norleamericana con el social-liberalismo que, en los países europeos, sigue denominándose impropiamęnte (en parte por economía del lenguaje y en parle, por motivos clectoralislas) "socialdemocracia”.

El planteamiento de Dworkin deja abicrtas algunats dudas e interrogantes. Enumeraré algunas que han sido sido subrayadas en la discusión de las reseñas de esile libro.

En primer lugar hay dudas fundadas de que pueda aceptarse su justificación del paternalismo para algunos casos. En segundo lugar, callo preguntarse hassa qué punto es realmente posible el igualitarismo político sin igualdad económica y si cabe admitir la preeminencia daclat a la igualdad de recursos independientemente del bienestar. Esto lleval a una terecral cuestion: ¿No es un limite del liberalismo actual, incluido el liberalismo élico de Dworkin, el dejar de plantearse la pregunta acercal del modelo social y político que se tiene in mente.

()tro posible tema de discusión liene que ver con la distinción que IDworkin establece entre "modelo de impacto" y "modelo de desafio". Y en ese ámbito parece que no está de más preguntarse si no cabe una versión. por así decirlo, débil de lo que Dworkin Ilama el modelo de impacto, tal que, deliniendo la vida huena como resultado de una aporlación valiosal a la humanidad sufricmese deje de presuponer o implicar que el fin justifica los medios. () sea. una versión debilitada, pero no estrictamente relativista, del modelo de impacio. i. Oueda resuclto el problema anterior mediante una concepción de la justicia que se articula en torno a la distribución de los recursos'? Y, todavía mais: ¿debemos incluir también la distribución intergeneracional de recursos, de acuerdo con una élica medioambiental, aunque sólo séa una ética antropocéntrical amplialal? ¿No nicga la elección del modelo (lel (lesalio, desarrollado por Dworkin para cerrar el hiato entre liberalismo ético y liberalismo politico, la posibilidad misma de otra versión de lo político como étical de lo colectivo, la que fue característica del pensamiento utópico desarrollado desde el Renacimiento y la Ilusiración”

\section{Bibi.iografía}

A. Heller, Más allá de la justicia, Barcelona, ('rítica, 1982.

F. Fernández. Bucy, "Crítical de una democracia demediada", en Ni trihunos. Madrid, Siglo XXI, 1906, cap. 2.

Hans Moravec, El hombre mécánico. I:I futuro de la robótica y la imeligencia humana. Barcelonia, Sallvall. 19(9);

Javier de Lucas, Puertas que se cierran. Barcelona, Icaria, 1990) Ipar la limilación actual del derecho de ciudadanial

Neil Postman, Tecnópolis, Barcelona, Círculo de Lectores, 1994. 
N. Chomsky, El miedo a la democracia. Barcelona, Crítica, 1992 Ipara la critica de la democraia realmente existente|.

J. Berger, Páginas de la herida. Madrid, Visor, I990) Ipara la cila sobre la democracia como una forma de resistencial

J. R. Capella, Los ciudadanos sicrvos. Madrid, Trotta, 1994, cap. II Ipar la crítica de la concepción proceclimentalistal.

J.M. González y F. Quesada (Coor.). Teorias de la democracia. Barcelona, Anthropos, 1988.

J. Rawls, El liberalismo politico |1993| (Iraclucción castellana: Barcelona, Critica, 1996.

R. Dworkin, Élica privada e igualitarismo politico. Barcelona, Paiclós, 199.3,

Serge-Christophe Kolm: Le's ćlections, sont-elle's la démocratie? París, İlitions du Cerl, 1979.

Ciudadonos y siervos: El "hombre mecónico", lo justicio y lo democrocio 